\title{
Nomination (Tasmiya) (Les rituels de la nomination dans le Sud marocain)
}

\section{Marie-Luce Gélard}

\section{(2) OpenEdition}

12 Journals

\section{Édition électronique}

URL : https://journals.openedition.org/encyclopedieberbere/2757

DOI : $10.4000 /$ encyclopedieberbere. 2757

ISSN : 2262-7197

\section{Éditeur}

Peeters Publishers

\section{Édition imprimée}

Date de publication : 5 octobre 2012

Pagination : 5616-5620

ISBN : 978-90-429-2718-6

ISSN : 1015-7344

Référence électronique

Marie-Luce Gélard, « Nomination (Tasmiya) (Les rituels de la nomination dans le Sud marocain) », Encyclopédie berbère [En ligne], 34 | 2012, document N68, mis en ligne le 15 décembre 2020, consulté le 17 février 2022. URL : http://journals.openedition.org/encyclopedieberbere/2757 ; DOI : https:// doi.org/10.4000/encyclopedieberbere. 2757

Ce document a été généré automatiquement le 17 février 2022.

(c) Tous droits réservés 


\title{
Nomination (Tasmiya) (Les rituels de la nomination dans le Sud marocain)
}

\author{
Marie-Luce Gélard
}

1 Comme dans toute société, les rituels de la naissance sont nombreux et complexes, ils marquent l'acquisition par l'enfant du statut d'individu. Seuls ces rituels et leur succession ordonnée (isolement, protection, soins du corps, maquillage, pratiques galactogènes, confection et pose d'amulettes, etc.) permettent au nourrisson de naître à la société. Parmi eux, celui de l'imposition du nom (tasmiya, en arabe ${ }^{1}$ ) est capital : il est un premier rite de passage spécifique grâce auquel le nourrisson acquiert une identité nominative propre. En effet, durant les sept premiers jours l'enfant ne dispose pas de prénom. Pour le désigner différentes formules ou métaphores sont utilisées, comme : « celui/celle qui est né(e) », « celui/celle que l'on félicite », etc. Il est aussi fait mention du prénom et du nom de sa mère. La filiation du nourrisson est exclusivement utérine, il n'est fait référence ni au père ni à son nom. La semaine qui suit la naissance se caractérise par son environnement exclusivement féminin. Dans la plupart des tribus du Sud marocain ${ }^{2}$, et plus particulièrement chez les Aït Atta*, la confusion entre le corps du nourrisson et celui de sa mère est entretenu par la similitude du maquillage sourcilier, de la coiffure (bandeau frontal), du henné apposé sur l'ensemble des mains et des pieds et non exclusivement sur la paume et la plante. L'établissement d'une ressemblance des corps (Gélard 2005) est destinée à protéger le nourrisson des tentatives de préemption par les êtres néfastes ${ }^{3}$. Dans l'oasis de Tabelbala ${ }^{4}$, la confusion est pareillement entretenue, pour tromper les jnûn et l'ogresse (tamza) on dépose une coloquinte à proximité de la tête de l'enfant qui agit comme un leurre (Champault, p. 318).

2 La subrogation de l'enfant ${ }^{5}$ (wanna ittubeddaln, littéralement: «celui [l'enfant] qui est échangé) est indiscernable car l'apparence du nourrisson demeure inchangée. On ne prend conscience de la substitution qu'en constatant un déficit de croissance souvent matérialisé, plus tard, par un handicap physique. Ces enfants sont connus pour ne vivre 
que peu d'années. En général, un enfant ne peut être changé au-delà du septième jour, c'est-à-dire après sa nomination.

3 Au matin du septième jour, la nomination de l'enfant va marquer la fin de la similitude mère/nourrisson, désormais ce dernier est devenu un individu, une personne.

\section{Sacrifice, nomination et morphologie prénominale (Cf. Gélard 2007)}

Le sacrifice réalisé pour la naissance d'un enfant est une recommandation religieuse et non une obligation. L'intervention des hommes ne débute au sein des différents rituels de la naissance qu'à l'occasion de ce sacrifice, lorsque la famille proclame: «Nous lui avons donné un nom » (nga-yas ism). Après la première prière, le père de l'enfant égorge un mouton en présence de toute la famille, de la mère et de son nourrisson.

Pour la première fois, la mère se lève et peut aller à l'extérieur. Le plus souvent, lorsque le père pose le couteau sur la gorge de l'animal, c'est la grand-mère paternelle ou un enfant qui prononce le prénom du nourrisson. Le père le répète et l'associe au patronyme. Les prénoms sont fréquemment choisis par les femmes, grand-mère ou mère du nourrisson ${ }^{6}$. Les cérémonies de nomination sont doublement signifiantes, d'un point de vue strictement rituel et statutaire mais aussi d'un point de vue politique et apparaissant comme le reflet de traits spécifiques à l'organisation sociale. Ainsi, la morphologie prénominale (dans les villages sahariens de l'extrême sud-est marocain) s'établit selon trois orientations : la dimension religieuse, la destinée familiale et la revendication identitaire. La plupart des Berbères marocains utilisent des prénoms arabes, ceux faisant référence au Prophète et à son proche entourage : Aïcha, Ali, Brahim, Fatima, Khadija, Mohamed, etc. Par contre, les prénoms issus de la culture arabe sont quasiment absents comme Abdellah, Jamila, Leila, Marouane, Naïma, Samira, Sifdine, etc. Les prénoms arabes sont donc choisis en référence à l'islam et destinés à protéger les individus qui les portent en vertu de la baraka* qu'ils leur confèrent.

Ces prénoms sont parfois supplantés par d'autres en fonction de la configuration de la fratrie et de l'histoire familiale. Ainsi, dans des régions où la mortalité infantile demeure élevée, de nombreuses familles nomment le garçon né après le décès de plusieurs enfants : Idir, prénom berbère signifiant littéralement « [qu'] il vive ${ }^{7}$ »! (Tidir pour les filles). Lorsque le prénom Idir ne peut être choisi, en général parce que le père le porte déjà, il est remplacé par M'barek dérivé de l'arabe baraka permettant ainsi de protéger la vie de l'enfant. Et enfin, une orientation récente dans le choix des prénoms est celle de la revendication identitaire conduisant à la réapparition de prénoms berbères. Si la génération des plus anciens porte le plus souvent des prénoms berbères comme Addi, Amu, Amrouch, Bedda, Herru, Itțo, Izza, Mamma, Mohda, Tudert, les trentenaires ont plutôt des prénoms arabes. Or, aujourd'hui, les prénoms berbères sont de plus en plus choisis et marquent une forme d'opposition symbolique à la culture arabe vécue comme dominante. Certains inventent même de nouvelles appellations comme Ayyur (prénom masculin signifiant «lune»). Traditionnellement les prénoms berbères font référence à une qualité humaine comme le courage, et non à des éléments ou à des objets. Ces choix récents sont critiqués par les anciens et peuvent donner lieu à des conflits familiaux. De plus, on relève aujourd'hui l'apparition d'un nouveau prénom arabe Belkassem (Belqasem), choisi en référence explicite à un célèbre 
opposant au pouvoir du Makhzen et au colonisateur français au début du XXe siècle. Belkassem Ngadi, d'origine idrisside se réfugia au Tafilalt et combattit auprès des opposants berbères Ait Khebbach.

7 Ces quelques éléments d'anthroponymie indiquent et révèlent la nature des relations entre groupes. Le choix des prénoms devient un enjeu politique de taille face à la revendication identitaire. En effet, de nombreux cas d'interdiction de prénoms berbères (Simane, Idir) par les officiers d'état-civil sont relayés par la presse et les sites internet ${ }^{8}$.

Une fois le sacrifice réalisé, toute la journée, parents et amis sont conviés au repas. Chez les Aït Hadiddou du Haut Atlas oriental, le repas de midi est réservé aux femmes du village (ahrir $n$ wudi d imryan). Le repas du soir est offert à tous les hommes du village (sksu d imryan d tutliwin) ${ }^{9}$ (D. Azdoud, information orale, avril 2011).

9 La mère et l'enfant sont entourés des femmes qui chantent et dansent sans discontinuer. Les hommes de la parenté et du voisinage mangent ensemble et félicitent le père. Dès lors, l'agrégation de l'enfant à sa famille paternelle est réalisée. La similitude mère-enfant établie par le maquillage et la coiffure disparaît; seul l'enfant porte encore le bandeau frontal, alors que la mère a retiré le sien après le sacrifice.

10 C'est bien le rituel sacrificiel associé au repas pris en commun qui marque l'entrée de l'enfant dans le groupe paternel : «[...] C'est à ce moment-là seulement que l'enfant naît à la famille, à la société (même s'il a déjà un nom), car jusque-là il était “inexistant" » (Aubaile-Sallenave 1999, p. 160).

11 Le rituel de la nomination va doublement signifier l'humanisation du nourrisson car outre l'attribution d'un nom, c'est la première fois qu'il est habillé de vêtements et emmailloté. Selon Nicole Belmont (1989, p. 11) l'emmaillotement aurait pour origine la volonté d'humaniser les nourrissons en les contraignant à étendre leurs jambes afin qu'ils ne marchent pas plus tard à quatre pattes comme les animaux. Pour les Aït Atta, cette technique est destinée à rendre plus forts les nourrissons en développant leur masse musculaire. L'enfant, empêché de tout mouvement, force sur ses membres afin de se libérer et ainsi se muscle. Le procédé favorise aussi le transport du nourrisson. En effet, compte tenu de sa petite taille, il ne peut être placé à califourchon sur le dos de sa mère. Il est donc emmailloté et placé en travers puis maintenu par un large morceau d'étoffe que la mère noue sur sa poitrine. Ainsi, habillement et emmaillotement au jour de la nomination signifient pour l'enfant sa naissance à la société : il est une personne. En principe, le lendemain, sa mère part chez ses parents jusqu'au matin du quarantième jour, pour une période de repos ${ }^{10}$.

12 Le septième jour est une cérémonie capitale à laquelle cependant tous les nouveau-nés n'ont pas droit. En effet, les enfants nés hors mariage en sont privés. Les enfants adultérins ne portent jamais le nom de leur père, même lorsque ce dernier est connu de tous, et sont ainsi stigmatisés. Dans ces cas, il n'y a pas de rituel de nomination et l'enfant porte le prénom de sa mère en guise de patronyme. Au final, seule la célébration de la tasmiya permet à l'enfant d'obtenir le nom du père, affiliation reconnue et légitime.

13 La tasmiya accomplie, l'enfant dispose donc d'une identité nominative propre, il devient pleinement une personne et cette attribution est significative de la reconnaissance collective de l'individu.

$14 \rightarrow$ Voir aussi « Naissance », « Onomastique ». 


\section{BIBLIOGRAPHIE}

AUBAILE-SALLENAVE Françoise, 1999 - «Les rituels de la naissance dans le monde musulman », in Pierre BONTE, Anne-Marie BRISEBARRE, Altan GOKALP [eds.], Sacrifices en islam. Espaces et temps d'un rituel, Paris, CNRS, p. 125-160.

BELMONT Nicole, 1971 - Les signes de la naissance, Paris, Plon.

BELMONT Nicole, 1973 - « Levana, ou comment élever les enfants », Annales E.S.C., p. 77-89.

BELMONT Nicole, 1980 - « L'enfant exposé », Anthropologie et sociétés (4-2), p. 1-17.

BELMONT Nicole, 1988 - «L'enfant et le fromage », L'Homme (105), p. 13-28.

CElAmPault Dominique, 1969 - Une oasis du Sahara nord-occidental. Tabelbala, Paris.

GAUDRY Mathéa, [1929] 1998 - La femme chaouia de l'Aurès, Paris, Chihab-Awal.

GÉLARD, Marie-Luce, 2003 - « De la naissance au septième jour. Rituels féminins et temps suspendu (tribu berbérophone du Sud-Est marocain », Ethnologie Française, XXXIII, p. 131-139.

GÉLARD, Marie-Luce, 2007, « De soi à l'autre. Approches du système de dénomination au sein d'une tribu saharienne (Aït Khebbach) ", Langage et société, 119, p. 161-182

\section{NOTES}

1. En berbère, on utilise l'expression à noyau verbal əg isəm (phonologiquement /g ism/), « donner (un) nom »; le concept n'est pas lexicalement nominalisé, d'où l'emploi de l'emprunt arabe tasmiya, " dénomination », facilité par la ressemblance formelle, la racine chamito-sémitique $S M$, «nom » étant à la fois berbère et arabe (isəm, «nom », n'est absolument pas un emprunt du berbère à l'arabe, contrairement à ce que l'on peut lire çà et là). NDLR.

2. Je remercie vivement Driss Azdoud pour les informations concernant les rituels de la nomination dans le Haut Atlas oriental.

3. Les jnûn tentent d'échanger l'enfant contre un des leurs, c'est le motif itératif du changeling, croyance en l'enfant changé que l'on retrouve dans de nombreuses sociétés, voir les descriptions de N. Belmont à propos de l'Europe.

4. Lieu de nomadisation des tribus Aït Atta et particulièrement des Aït Khebbach.

5. Également relevé au Maroc par la doctoresse Legey (1926, p. 85). «Lorsque la toilette de l'enfant est terminée, la sage-femme le passe trois fois à la droite de la mère, puis à sa gauche, en disant: "Que tu sois à droite ou à gauche, sois sans crainte, les diables ne t'emmèneront pas" ". Ainsi qu'en Algérie (Aurès) par Mathéa Gaudry (1998, p. 110-111).

6. D'après Driss Azdoud (information orale, avril 2011), chez les Aït Hadiddou du Haut-Atlas oriental, un peu avant la naissance, la famille restreinte (le père, la mère, et les deux grandspères en priorité) fait des propositions de prénoms. La veille du sacrifice, le choix est arrêté. C'est le fqih du village qui est invité à égorger le mouton. Il prononce la formule suivante : nz̧zull xf nnbi

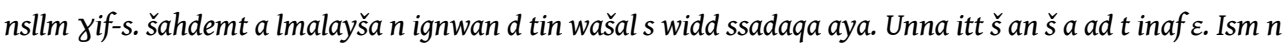
le il/trbat $n$ flan X/Y.

7. Il s'agit de la $3^{\mathrm{e}}$ pers. du sing. ( « il ») du thème d'aoriste du verbe idir/dder, « vivre ». L'aoriste a fondamentalement valeur modale, ici optative (cf. S. Chaker \& A. Mettouchi : « Mode/modalité », EB XXXII). NDLR.

8. www.mondeberbere.com et amazighworld.net 
9. Tagine pour les femmes, couscous et brochettes pour les hommes. Le repas est légèrement plus salé qu'à l'accoutumée. Dans la journée, on fait venir une personne du village, connue pour avoir les plus beaux traits du visage, elle est munie de babouches neuves et blanches qu'elle porte aux mains comme des gants. Elle les utilise pour porter le bébé sans le toucher et le poser sur le dos de sa mère. (D. Azdoud, information orale).

10. Cette phase correspond à la période de post-partum telle qu'elle est définie par les médecines occidentales, soit environ six semaines après l'accouchement. L'OMS définit le post-partum comme une période allant de 28 à 40 jours après la naissance.

INDEX

Mots-clés : Ethnologie, Maroc, Maroc central (zone tamazight) 